\title{
Frontiers
}

\section{in the Psychotherapy} of Trauma \& Dissociation

The Official Clinical Journal of the International Society for the Study of Trauma and Dissociation
EMDR for Safe Desensitization of Memories and Fusion of Parts in DID. Conference Room Technique, Trauma Mapping and Management of Unplanned Abreactions Neva Shebini, MBBS BSc (Hons) MRCPsych FRANZCP
International Society for the Study of Trauma and Dissociation 


\title{
Frontiers in the Psychotherapy of Trauma \& Dissociation
}

The Official Clinical Journal of the ISSTD

\section{EDITOR}

ANDREAS LADDIS, MD, Private Practice and Faculty, Boston University, School of Public Health, Boston, Massachusetts, USA

\author{
ASSOCIATE EDITOR \\ MARTIN J DORAHY, PhD, Professor, Department of Psychology, University of Canterbury, \\ Christchurch, New Zealand and The Cannan Institute, Brisbane, Australia
}

\section{EDITORIAL ASSISTANT}

COURTENAY CRUCIL, MA, RCC, Private Practice, Terrace, British Columbia, Canada

\begin{abstract}
Frontiers in the Psychotherapy of Trauma \& Dissociation is published by the International Society for the Study of Trauma and Dissociation, Inc., 1420 New York Ave NW, Fifth Floor, Washington, DC 20005.
\end{abstract}

\section{Annual Subscription, Volume 3, 2019}

Online subscription is part of the membership dues of the International Society for the Study of Trauma and Dissociation. Visit https://www.isst-d.org/join-isstd/individualmember-benefits/.

Production and Advertising Office: ISSTD Headquarters, 1420 New York Ave NW, Fifth Floor, Washington, DC 20005.

Copyright @2019 International Society for the Study of Trauma and Dissociation. All rights reserved. No part of this publication may be reproduced, stored, transmitted, or disseminated in any for or by any means without prior written permission from the International Society for the Study of Trauma and Dissociation. The publisher assumes no responsibility for any statements of fact or opinion expressed in the published papers. The appearance of advertising in this journal does not constitute an endorsement or approval by the publisher, the editor, the editorial board, or the board of directors of the International Society for the Study of Trauma and Dissociation of the quality or value of the product advertised or of the claims made of it by its manufacturer.

Subscriptions to this journal are acquired through membership in the International Society for the Study of Trauma and Dissociation only.

Visit https://www.isst-d.org/join-isstd/individual-memberhip-categories/.

Permissions. For further information, please write to info@isst-d.org.

\section{EDITORIAL BOARD}

ELIZABETH S BOWMAN, MD, Editor Emerita, Journal of Trauma \& Dissociation, Adjunct Professor of Neurology, Indiana University School of Medicine, Indianapolis, Indiana, USA

LAURA S. BROWN, PhD, Private Practice, Seattle, Washington, USA

RICHARD A CHEFETZ, MD, Private Practice, Faculty and Founding Member Institute of Contemporary Psychotherapy \& Psychoanalysis, Distinguished Visiting Lecturer: William Alanson White Institute of Psychiatry, Psychoanalysis \& Psychology, New York City, USA

CONSTANCE J DALENBERG, PhD, Trauma Research Institute, California School of Professional Psychology, San Diego, California, USA

J.K. JUDITH DANIELS, PhD, Faculty of Behavioural and Social Sciences, University of Groningen, The Netherlands

STEVEN N GOLD, PhD, Professor, Center for Psychological Studies, and Founding Director, Trauma Resolution \& Integration Program, Nova Southeastern University, Fort Lauderdale, Florida, USA ELIZABETH B HEGEMAN, PhD, Professor, Department of Anthropology, John Jay College of Criminal Justice, New York, New York, USA 
RICHARD P. KLUFT, MD, PhD Private Practice and Clinical Professor of Psychiatry, Temple University School of Medicine; Faculty Member, Philadelphia Center for Psychoanalysis,

Philadelphia, Pennsylvania, USA

CHRISTA KRÜGER, MD, Professor of Psychiatry, University of Pretoria, Pretoria, Gauteng, South Africa

KARLEN LYONS-RUTH, PhD, Professor of Psychology, Harvard Medical School, Cambridge, Massachusetts, USA

ALFONSO MARTÍNEZ-TABOAS, PhD, Professor, Albizu University, San Juan, Puerto Rico WARWICK MIDDLETON, MD, Adjunct Professor, Cannan Institute, Brisbane, Australia

ELLERT R. S. NIJENHUIS, PhD, Department of Psychiatry and Outpatient Department Mental Health Care Drenthe, Assen, The Netherlands

SANDRA PAULSEN, PhD, Bainbridge Institute for Integrative Psychology, Bainbridge Island, Washington, USA

VEDAT SAR, MD, Professor of Psychiatry, Koç University School of Medicine (KUSOM), Istanbul, Turkey

JOYANNA SILBERG, PhD, Trauma Disorders Program, Sheppard Pratt Health Systems, Baltimore, Maryland, USA

ELI SOMER, PhD, Professor, School of Social Work, University of Haifa, Israel

KATHY STEELE, MN, CS, Private Practice, Atlanta, Georgia, USA

ONNO VAN DER HART, PhD Emeritus Professor of Psychopathology of Chronic Traumatization, Department of Clinical and Health Psychology, Utrecht University, Utrecht, The Netherlands VICTOR WELZANT, PsyD, Sheppard Pratt Health Systems, Trauma Disorders Program

\section{REVIEWERS}

JOHN BRIERE, PhD, Associate Professor of Psychiatry and Psychology, University of Southern California Keck School of Medicine, Los Angeles, California, USA

SHELDON IZKOWITZ, PhD, Clinical Associate Professor of Psychology and Clinical Consultant, Postdoctoral Program, New York University, New York City, USA and Teaching Faculty \& Supervisor of Psychotherapy and Psychoanalysis, National Institute for Psychotherapies, New York City, USA

MARY-ANNE KATE, PhD Researcher at University of New England, Australia; University of New England, New South Wales, Australia

ULRICH F. LANIUS, PhD, Private Practice, West Vancouver, British Columbia, Canada

\section{SUPPORTERS}

ISSTD thanks its generous supporters whose contributions have made this publication possible:

Andreas Laddis, MD, USA

\section{The \\ Cannan \\ Institute}

Cannan Institute, Australia

Warwick Middleton, MD, Australia

Dana Ross, MD, Canada

Martin J. Dorahy, PhD, New Zealand

Kate McMaugh, Australia

Sara Y. Krakauer, USA

Paula Eagle, MD, USA 


\title{
ARTICLE
}

\section{EMDR FOR SAFE DESENSITIZATION OF MEMORIES AND FUSION OF PARTS IN DID. CONFERENCE ROOM TECHNIQUE, TRAUMA MAPPING AND MANAGEMENT OF UNPLANNED ABREACTIONS}

\author{
NEVA SHEBINI, MBBS BSc (Hons) MRCPsych FRANZCP \\ Consultant Psychiatrist
}

This paper explores the use of Eye Movement Desensitization and Reprocessing therapy (EMDR) in the treatment of Dissociative Identity Disorder (DID), in its most severe form. I discuss suggested modifications and applications for the safe use of EMDR, including the essential use of the conference room technique. I have described the concept of "trauma memory mapping" before EMDR is delivered. Additionally, this paper considers how parts may be created and the possible connections between parts within a trauma memory. Trauma memory desensitization, and the associated fusion of parts into the system, with the planned use of $E M D R$ is described. Insights from the management of internal resistance to fusion are presented. A case example with safe management of an unplanned abreaction has been provided. A mention is given to the comparison of use between hypnosis facilitated abreaction and EMDR.

KEYWORDS EMDR; dissociative identity disorder; satanic ritual abuse; tertiary structural dissociation; child parts modifications; trauma memory desensitization and reprocessing; trauma memory mapping; conference room technique; fusion of parts; abreaction in EMDR; hypnosis; psychiatry; psychotherapy

Acknowledgements: Thank you to Bessel Van der Kolk for his support and wisdom and encouraging me to write this up. Thanks to my client who has made all of this possible.

Author Contact Information - Private Practice, Peace of Mind Services Ltd., Cairns, Queensland, Australia, Tel. +61 0740310419, email: Info@peaceofmindservices.com.au. 
This is the first in a series of two papers on the subject of trauma memory processing and the planned fusion of parts, in a case of extreme Dissociative Identity Disorder (DID) from satanic ritual abuse. This paper focuses on the use of Eye Movement Desensitization and Reprocessing (EMDR; Shapiro, 1995, 2018) and the second paper focuses on the use of the Flash technique (Manfield, Lovett, Engel \& Manfield, 2017).

I believe that there is an underuse of EMDR in the treatment of dissociative disorders. This may be due to a lack of training in EMDR, therapist preferences to use other interventions for trauma processing or a fear for therapists that EMDR could destabilize their already unstable clients. Alternatively, therapists may have basic training in EMDR but do not feel "skilled enough" to use it with these clients. I hope to shed some light and interest for therapists, both new and familiar in this field, on this exciting and invaluable therapy tool. This paper pays particular attention to the possible modifications and the safe application of EMDR therapy for complex trauma and dissociative disorder clients. I hope to encourage therapists who are new to EMDR to train in and ultimately use this therapy. I also hope that therapists who are currently trained in EMDR can learn effective modifications, making it suitable for use with their complex trauma and DID clients.

\section{PHASE-ORIENTED TREATMENT APPROACH}

The recommended treatment for complex trauma disorders is the phaseoriented treatment approach (International Society for the Study of Trauma \& Dissociation [ISSTD], 2011), which originates from Pierre Janet (1889) in the late 19th century. These phases are usually not linear; the client will need to return back to earlier phases intermittently throughout their therapy. The phases are:

1. Stabilization and symptom reduction;

2. Confronting, working through and integration of traumatic memories;

3. Identity integration and rehabilitation.

Traditionally in phase 2, traumatic memories have often been treated using hypnosis and a psychodynamic approach. These methods are still important, however, treatment can be significantly faster with the use of early screening for dissociation, Fraser's (1991) Dissociative Table Technique and EMDR (Paulsen, 2008). I recommend intertwining EMDR with other psychotherapies during phase 2, for treatment of complex trauma and DID. 


\section{DISSOCIATIVE IDENTITY DISORDER}

DID is not an uncommon condition. A collective study of psychiatric inpatients, outpatients and chemical dependency patients revealed that $4 \%$ to $18.6 \%$ had a missed diagnosis of DID (Kluft, 1999). The theory of structural dissociation of the personality (TSDP) is useful for understanding and treating the disorders of traumatization, including DID (Van der Hart, Nijenhuis, \& Steele, 2006; Steele, Boon \& Hart, 2017). The TSDP describes the personality of traumatized individuals as being made up of dissociative parts, which can be categorized as Apparently Normal Parts (ANPs) and Emotional Parts (EPs). Tertiary structural dissociation often involves several ANPs and many, if not hundreds of EPs, as in the case presented in this paper. The ANPs serve to function in everyday life with reduced awareness of their past experiences. The EPs carry the different elements of traumatic material including memory, affect, somatic experiences and cognitions. For the purposes of this material, I will be referring to the main personality presenting for treatment as the "host." I will use the general term "parts" when referring to Eps, and I will continue to use the term "ANP" for ANPs other than the host.

\section{BACKGROUND}

I have been working extensively with a highly fragmented client with an elaborate personality system, who reported horrific abuse in a satanic cult over many years. She has a diagnosis of DID with disabling posttraumatic stress disorder. I have now worked with her for six years and see her six times per month. She was in the mental health system for 10 years prior to working with me and had made little progress; in fact, she became retraumatized by psychiatrists and mental health staff who misunderstood her condition. Her presentation was frequently in the emergency department for repeated severe self-mutilation and suicide attempts. She was labeled as a "difficult borderline" who was "a waste of time and resources," and she was often prescribed heavy doses of medication to sedate her.

Very few mental health professionals have had any formal training in the assessment and treatment of DID, and as a psychiatrist, this condition was extremely new for me. Consideration was given to moving this client on to a more suitable and experienced therapist, however, there were none in the region. This led me to do a significant amount of professional development in the areas of trauma, dissociation and DID, which has allowed me to help this client and other complex trauma sufferers. Only after having adequate training, sound knowledge, regular supervision and clinical experience with a number of "simpler" clients, can one then consider working with such complex presentations. 


\section{EMDR THERAPY AND MODIFICATIONS}

EMDR is usually delivered with a standard protocol consisting of eight phases (Shapiro, 1995, 2018). However, controversy regarding the use of EMDR in dissociative disorders has continued since the first developments in EMDR therapy. A task force for the treatment of dissociative disorders recommended "extreme caution" (EMDR Dissociative Disorder Task Force, 1994). To date there is no specific and validated standard protocol for DID clients (Ross, 2015). I would like to suggest the following EMDR adaptations for DID.

\section{The Conference Room Technique (Fraser, 1991)}

The Conference Room Technique is a powerful tool that allows parts of the self to safely come together in one place that is "shut off" from the rest of the system. Parts that are outside of the conference room cannot "hear" any of the therapy discussions occurring inside the room, hence they can be protected from unnecessary exposure to other parts' trauma experiences or crises that may be occurring. I have found it invaluable to use particularly with the unexpected emergence of "new" parts that are commonly disorientated, re-living their trauma and using self-harm as a rapid attempt to cope with their distress. Psychoeducation and grounding can occur quickly with the host or helper ANPs assisting within the conference room. The therapist guides this safety work, but over time, the client should learn to use the conference room outside of therapy sessions, for issues that arise with parts. With a little practice, the client can set up the conference room in a mere second or two, and invite selected parts in.

My client's conference room has a clever set up. The main room has comfortable lounging chairs for the parts to sit on, and they can all "look through the eyes" to see and hear the therapist, on the outside. The walls are decorated with rainbows and other attractive art. There is a waiting room attached where parts can "wait." It is a soundproof room, but there is a speaker that can be turned on, allowing the option for parts in the waiting room to hear what is being discussed in the conference room, but not see. The waiting room also has headphones with calming music, if parts chose to use that resource. There is also another soundproof room attached, the library, where memories can be filed away in filing cabinets between sessions. Also in the library and the main conference room are decorated boxes (designed by the parts) used for traumatic memory containment.

\section{Trauma Memory Mapping and Internal Self Helpers}

I recommend trauma mapping in DID as an important strategy that can be used to facilitate the guiding of the therapy, however, it may not always be possible to achieve. Trauma mapping can be seen as an extension of 
personality system mapping, which was first discussed by Morton Prince (1909) and Walter Prince (1917). They published diagrams of how they thought a patient's parts fit together, which could then help to identify which parts would likely be able to fuse easily together during the series of partial fusions that build towards the final fusion.

It can be written down or drawn and is similar to the concept of looking at a city's underground subway system. One of the most important aims of trauma mapping is to avoid unplanned abreactions from unmapped parts that emerge in the middle of trauma therapy. That part may have no idea that trauma therapy is taking place and is then made to re-live their trauma through exposure during the EMDR. This can shock the other parts and distress the whole system. Therapeutic rapport can be damaged and repair work may need to take place. Another use of trauma mapping is to assist the therapist in selection of the target memory.

It is extremely helpful to have a strong alliance with the parts that know the most about their trauma experiences (Internal Self Helpers [ISH]), and they can help the therapist to map them out. It is not clear if they are present in all DID patients but thought to occur in most. Some patients may have more than one ISH. I was fortunate enough to have been greatly helped by an ISH, an older ANP called "W" who had a role to remember everything that ever happened to them. She had an impressive internal detailed log of traumas and remembered which parts sustained which trauma and the specifics of each trauma memory. She was able to map this out on paper in several different formats including lists and mind maps. There was a "hierarchical" map of how the all parts were linked and related together within the system. Additionally, there was a map that displayed the individual trauma memory experiences and which parts were created or involved in those events. Using the maps, we formulated a strategy plan which involved the targeting of traumas that were chronologically experienced from young ages first, starting from under the age of one year old.

\section{Target Memory}

In dissociative disorders, I would recommend caution if using the affect scan technique (Shapiro, 1995) as this may trigger further dissociation and destabilize the client. The phobia of traumatic memory and peripheral memories should be targeted and overcome first, before approaching the more core memories of their traumatic experience. The progressive approach (Gonzalez \& Mosquera, 2012) involves the processing of experiences that are related to the trauma, in a very gradual way, before working directly with the memory itself. If trauma mapping is possible, it can guide the path of the therapy. 


\section{Fractionation}

Fractionation is the dividing up of memories into fragments. For some patients, a fractionated abreaction procedure can be used to dilute the intensity of the affect and prevent re-traumatization (Kluft, 1988).

\section{Negative Cognition (NC) / Positive Cognition (PC) / Validity of Cognition (VoC) / Subjective Units of Distress (SUDS)}

In DID, the therapist is often working with young child parts who demonstrate an apparent difficulty in understanding ideas and language and may differ in their ability to think abstractly, some of them being very concrete. These parts have limited or no language to describe emotions such as fear, anger, betrayal, disgust and shame. They may not be able to reveal a NC, PC, VoC or SUDS. In addition, insisting to obtain these elements may "front load," whereby the part gets lost in analysis and takes them away from the network that we are trying to activate (Parnell, 2013). Hence, I believe that if necessary, these elements from the standard protocol can be omitted, particularly with parts under the age of six. This is consistent with EMDR protocols modified for children (De Roos, Beer, Jongh, \& Broeke, 2015). If the SUDS is omitted, another indication for the level of distress should be obtained.

\section{Process}

I have found that explanations on the process of EMDR can be kept basic, and with simple language, so that young parts can understand. Reassurance and encouraging statements during the therapy are very helpful for the parts. I advise that I will stop every so often (for example every 10 to 20 seconds) and ask them what they notice. They do not need to reveal specific details of what they are "seeing" and experiencing, unless they wish to. Cognitive interweaves are helpful if they become distressed or stuck. Emotional distancing techniques can be used to reduce distress and may include changing the memory into a still photo, a black and white video or using a protective glass wall between the part and the event.

\section{Desensitization and Reprocessing}

Trauma memories can be viewed as having three components: the sensory experience (images, smells, sounds, taste and touch), the cognitive component, and the emotional and somatic experiences. Desensitization with EMDR means that there has been a reduction in the intensity of the memory. It becomes harder to recall the memory. The ideal aim is for reprocessing of traumatic memory, however, this may not always be possible. Reprocessing involves the complete desensitization of the trauma, as well as the essential cognitive restructuring. The installation phase installs and strengthens the 
PC, and the VoC rises to a seven. The body scan checks for residual emotions and sensations which are processed with further bilateral stimulation. The client can finally make sense of their traumatic experience. They can look back on it from a more neutral stance and give a coherent personalized narrative.

\section{Bilateral Stimulation (BLS)}

It is recommended to use slower and shorter sets (initially five to six sets) of BLS in response to the client's ability to process traumatic material. The length and speed of the EMDR sets should be changed to accommodate each client and also their parts. If they use bilateral handheld stimulators, they are advised that their eyes can be open or closed; caution should be taken if their eyes are closed as this could contribute to dissociation in some clients.

\section{HOW ARE PARTS RELATED IN A TRAUMA?}

Before undertaking EMDR, it is important to understand how parts are related in a trauma memory. More than one process may be involved in the separation of traumatic memory material into dysfunctionally stored fragments. When the experience of trauma is divided amongst several parts, one possibility is that each part may have experienced a time segment of the event. Each individual part would have their own Behavior, Affect, Sensation and Knowledge (BASK) model experiences (Braun, 1988a, $1988 \mathrm{~b})$. When parts follow each other chronologically in time, this is termed "sequential" or "linear" dissociation (Van der Hart, Steele, Boon, \& Brown, 1993). On the other hand, "parallel" or "simultaneous" dissociation occurs when parts experience the same traumatic experience, but they contain different aspects of it, per the BASK model. Emotional parts holding the experience of trauma can also be grouped together in clusters of families. These families may be related to the memory they share, or to the different components (affects, cognitions, sensations) that they share. Trauma mapping (see section above) can be used to display these "families." In this client's system, parts were predominantly clustered together according to the trauma event that they experienced.

This is invaluable knowledge before commencing EMDR, however, it may not always be possible to acquire such knowledge and detail. Parts in sequential dissociation are likely to replay their memories "in sequence" in an EMDR session. Each part can be selected to have their own EMDR session, or all the parts in one memory can be treated in the same session (see case example below). In sequential dissociation, there is likely to be a higher number of BASK channels to process. In linear dissociation, I would advise to give individual parts their own EMDR session. 


\section{A CASE EXAMPLE}

\section{Phase 1: Stabilization}

The first three years of working with my client was solely focused on phase 1: stabilization. This mainly involved building a strong therapeutic alliance, managing and reducing suicide risk, working through frequent crises ("putting out fires"), symptom stabilization and reduction, hospitalizations, the cautious use of medications, development of safety plans, working directly with parts, resourcing and containment strategies and developing co-consciousness within the system. In DID, this phase may take years, and some clients may stay in this phase throughout their therapy lifetime. The client had approximately 300 parts, and many of them appeared to be cult created and programmed with mind control techniques. Initially there was a high level of dissociation between the parts, and many of them lacked awareness that other parts existed. Persecutor parts battled to gain control and relentlessly tormented one another with physical torture sensations (electric shocks, head spinning, loud ringing in ears), terror, body memories, self-mutilation, suicide attempts and flooding with disturbing nightmares. Most of the parts were under the age of 10 with an average age of five years old. There were several ANPs, and the average age was 25 years old. An estimate was that the client had 1,000 trauma memory fragments. I wonder how long this could take to work on with traditional psychotherapies. I discussed the option of trialing EMDR with my client, and she was agreeable to try the method.

\section{The First EMDR Session}

We planned to start EMDR with a trauma memory that was "less intense." It involved three young parts; they can be called parts 1, 2 and 3. Consent was obtained from all parts involved before any processing of traumatic material commenced. Parts 1 and 2 had experienced several other traumas, however, part 3 had only experienced this trauma event; my hypothesis was that if part 3 had complete trauma desensitization, then part 3 could fuse with the personality system.

The host waited in the conference room waiting room, and the helper part $\mathrm{W}$ was at the front of the conference room and present in the therapy office with me. The three child parts were inside the conference room. The three parts could see me through part W's eyes and could hear me talking. Part W talked to them about what could happen with EMDR, and I gave a further simple explanation also. The client was agreeable to use an EMDR bilateral stimulation device with soft bilateral stimulation in the form of buzzing in each hand.

In this first session, once EMDR commenced, each part observed their trauma memory in sequence. At the time of the actual trauma event, once 
the trauma became too much, part 1 dissociated to part 2, and once it was too much for part 2, she dissociated into part 3 (i.e., sequential dissociation; Van der Hart et al., 1993). This played out exactly so in EMDR. Once part 3 's memory played through the first time, it returned and replayed straight back to part 1's memory, but then it played out a little faster to part 2 and then part 3's memory. The memory replayed four times, and by that fourth time, there was subjective and objective desensitization of the memory. They reported that the image was smaller, less vivid, two-dimensional, had less color and was distanced ("further away"). Their body sensations reduced significantly.

\section{FUSION}

For the fifth "replay" or "pass" of the memory fragment with EMDR, I invited part 3 to switch places with part W so that she was on the outside and present in the office with me. The other two parts and part $\mathrm{W}$ stayed inside the conference room. Part 3 then had further EMDR, and she was observing the remnants of her memory. She looked sleepy and calmer. As the EMDR continued (with the bilateral stimulators on), she came to the "end" of her memory fragment, and it finally desensitized to completion. I then observed there to be a rapid transition between part 3's sleepy eyes closing completely, and then her eyes opening and rolling upwards with only the white sclera of her eyes visible. She sat upright and still. There was an unusual calmness about her. Observing the client further, she remained motionless with her eyes rolled upwards. This phenomenon is termed a Spiegel eye roll and was first described by Spiegel in 1972. It was used as a test of hypnotizability and theorized to indicate a person's biologically based capacity for trance.

That transition appeared to be the complete desensitization of the memory fragment, allowing that part to fuse with the personality system. After around one minute I asked to speak to part $W$ who came back out into the therapy office with me. She said, "She's gone, she's gone inside." It was confirmed that part 3 was no longer in the conference room and had fused with the personality system.

This was a fantastic outcome, and it proved the hypothesis to be truethat fusion could occur in this scenario. Later in the session, I discussed what had occurred with the host (who had no knowledge of our session). She was pleased of the results and reported that she could feel a small change in somatic sensations in her chest. She found it hard to describe with words, but it was like a "fizzing" or "bubbly" feeling. It was a sensation of something "clicking" into place. She was happy to continue with further EMDR sessions to metabolize other traumas and allow for more fusion of parts into the system. 


\section{Preparing parts for Fusion}

Fusion refers to the joining of two or more parts with a complete loss of subjective separateness (ISSTD, 2011). Fusion is seen as the initial "compacting" process that leads to groundwork for integration. Integration is "more pervasive and thorough psychic restructuring" (Kluft, 1984, p. 24). Partial fusions may be deliberately performed as part of the therapeutic plan to reduce the number of parts and unify the patient (Putnam, 1989). Planned fusions can also be conducted if it is the request of the personality system (Paulsen, 1995). One can use the personality system map as a guide; parts who are next to each other on the map or share a common connection in the map's overall metaphor will fuse together more easily.

In the case of my client, the host likened her parts to Gummy Bears. She is the big Gummy Bear, and her parts are many small Gummy Bears. With internal dialogue, she told them that they were all part of one big Gummy Bear. Parts were told how important and valuable they were. They were informed that the end result is for all the small ones to join together with her and the system. Many parts were really tired of their trauma memories and were open to fusion. However, some parts were scared, apprehensive and did not really know what was going to happen to them. During the EMDR therapy, scared parts could use grounding objects that they could see, feel, hold or smell. These included a soft teddy bear, flowers, fidget toys and cushions supporting them in their chair. Here are some things young parts have said in session, before fusion:

"I'm scared."

"Will I disappear?"

"What's going to happen?"

"Where will I go?"

"But if I'm not here, I'm gonna miss out on stuff."

"If I'm not here, I won't be able to help her."

"Who will she talk to if I'm not here?" (Referring to another young part)

"Will I see my teddy bear again?" (Soft teddy in my office used for grounding)

\section{Resistance to Fusion}

Some parts resisted fusion. They did not want to believe that they were a part of the client or the system as a whole; they believed that they were their own person. Different techniques can be used to help parts to see that this is not the case. One way was to ask them what they remembered about their different birthdays or childhood memories, of which they did not have an answer. Another way was to show the part their reflection in the mirror, the client's ID card or the wedding ring on the client's finger. Once the part could see that she was (just) a part, she was reassured that both she and 
the host would feel better once they join. The part was helped to see that they would never need to remember bad memories again and they could finally rest. If further persuasion was needed, then older parts who had previously observed fusion (during EMDR) could talk with them and offer reassurance.

\section{Managing Unexpected Abreactions}

In one session of EMDR, there was an unexpected abreaction. The planned session was with four young child parts, who had been involved in sexual abuse, which included being filmed for child pornography material. Halfway through the session a nine-year-old part $(\mathrm{Y})$ unexpectedly and abruptly emerged. She was visibly distressed and the whole body became tensed with convulsion type movements. She had worsening body pain. I had worked with part $Y$ in the past, so was aware of her presence and her history, but she had not been mapped out as being part of this specific trauma memory. At some point during that apparent trauma episode, the body was punished with electricity, which triggered the emergence of part $\mathrm{Y}$, who had previous such punishments. She was re-living being electrocuted. In the earlier trauma mapping process, part $\mathrm{W}$ did not recall (and was not aware) that part $\mathrm{Y}$ had emerged temporarily, during that abuse memory.

In cases like this, one must not panic, although it can feel extremely challenging, when exposed to the uncontrolled and highly distressing emergence of parts who re-live torture experiences in front of their therapist. EMDR training always advises for the therapist to encourage the client to "keep going" and make it through the "other side," "to the end of the tunnel." I kept grounding the part with my voice and reminding her that she will get out of the tunnel soon. I increased the speed of the bilateral stimulation a little, which increases the speed that they "go through" their memory fragment. After a few minutes of EMDR with part Y, she completed "going through" that part of her memory, once. In this particular example, we used grounding and containment strategies for the emerged part $Y$ that had the abreaction, and I cautiously continued EMDR with the other four parts. Part $\mathrm{Y}$ was given EMDR with the Flash technique separately. All five parts were eventually able to process their trauma memories successfully.

After the session, perfectionist helper part $\mathrm{W}$ was horrified for making an "error" and was apologetic and felt guilty. She felt bad that she did not remember that part $Y$ was involved in that memory and blamed herself for this highly distressing abreaction. It is important to remember that no one is perfect. Reassurance was given to comfort her, and once again I thanked her for her amazing abilities to work with me and to have been able to map out their other traumas. The therapist should always be prepared for the occurrence of unplanned or uncontrolled abreactions and should have strategies and confidence to manage the situation. 


\section{EMDR Session Closure}

After each EMDR session, the host and system were debriefed as to what material was worked on, which parts were involved and if any fusions occurred. If a memory has not been completely processed, it is best to avoid giving too many details to the host, to prevent unnecessary flooding and also to allow the host to continue to perform their everyday functions. The patient is reminded that after the session, they can experience aftershocks, mini abreactions, flashbacks, intrusive imagery and traumatic nightmares. They can keep a note of these experiences and bring it to the next session. They are advised to use the necessary grounding and relaxation strategies in between sessions.

\section{Progress to Date}

After the success of the first session, we continued with further EMDR therapy. In the following 12 months, she had a further 50 EMDR sessions. Approximately 300 distressing trauma memory fragments have been metabolized, and subsequently over 100 child parts have been permanently fused with the personality system. The client frequently needed to return to phase 1 stabilization techniques, during phase 2 trauma therapy, to manage emotional dysregulation, intrusive symptoms, abreactions and the emergence of new parts.

\section{DISCUSSION}

\section{Modifications}

EMDR Modifications can be within the preparation phase, the assessment phase and the desensitization/reprocessing phase. I have found that in most sessions with young parts, it was virtually impossible to obtain the NC, PC, VoC and SUDS. The ideal aim is complete desensitization and reprocessing of the traumatic memory, however, this may not always be possible. Nevertheless, significant desensitization is helpful to reduce the volume of traumatic material that the client has to "carry."

\section{How Much Trauma Memory Processing to do?}

Therapists disagree on whether or not to abreact every major trauma. Some DID patients have so much trauma that it is probably not possible to go through all of them. However, EMDR abreaction of one "generic" episode of a specific type of repeated abuse often may serve to metabolize the dissociated material from a series of related traumas. 


\section{Fusion}

Fusion of parts can be a planned procedure or a byproduct of therapy. In a client with such a vast amount of trauma memories and hundreds of parts, fusion was a welcomed process that "lessened" the volume of material that she had to carry. Fusion of a part appeared to be a natural occurrence with EMDR, once the part's memory had completely desensitized.

\section{Comparing Hypnosis and EMDR}

There appear to be noteworthy benefits to using EMDR over hypnosis when working with highly fragmented DID clients. Firstly, EMDR can work on a higher "volume" of material with a faster speed of processing, in one session. Secondly, EMDR-produced abreactions are often less painful than hypnotically produced abreactions, so affect tolerance is less of a problem (Paulsen, 1995). Third, EMDR has a lovely element, which is that the client does not need to verbalize any details of their trauma, to the therapist. This prevents flooding and allows privacy for the client.

\section{Work Intensity and Abreactions}

Trauma memory processing using EMDR can be emotionally and mentally draining and challenging for both client and therapist. This is especially the case for an unplanned abreaction, which can instill panic and possibly helplessness in the therapist. Awareness of one's own internal experiences and self-regulation during the session is recommended. Self-care and regular supervision in between sessions is strongly advised. Therapists should have training in managing an unplanned abreaction before undergoing such intensive trauma work.

\section{CONCLUSIONS}

In conclusion, this paper is the first of a series of two papers where I have discussed the safe use of EMDR in the treatment of traumatic memory and the resultant fusion of parts in complex DID. The client presented in this paper was involved in ritualized cult abuse, however, the strategies in this paper can be applied to clients with non-ritual abuse trauma. I hope that the modifications, suggestions and strategies provided in this paper can be of some assistance to therapists when working with clients suffering with DID and complex trauma presentations. I would very much like to welcome any comments and feedback on the work presented in this paper.

In the upcoming second paper in this series, I will describe another method with case examples, the Flash technique, which has also been used successfully for trauma memory desensitization and fusion of parts. 


\section{REFERENCES}

Braun, B. G. (1988a). The BASK model of Dissociation. Dissociation, 1(1), 4-23.

Braun, B. G. (1988b). The BASK model of Dissociation. Dissociation, 1(2), 16-23.

De Roos, C., Beer, R., De Jongh, A., \& Ten Broeke, E. (2015). EMDR protocol for children and adolescents $0-18$ years old. www.ariannestruik.com

EMDR Dissociative Disorder Task Force. (1994). EMDR Dissociation Task Force position paper. Pacific Grove, CA: EMDR Institute.

Fraser, G. A. (1991). The dissociative table technique: A strategy for working with ego states in dissociative disorders and ego-state therapy. Dissociation, 4(4), 205-213.

Gonzalez, A., \& Mosquera, D. (2012). EMDR and dissociation: The progressive approach. Charleston, SC: A. I.

International Society for the Study of Trauma and Dissociation. (2011). Guidelines for treating dissociative identity disorder in adults, third revision. Journal of Trauma E Dissociation, 12(2), 115-187.

Janet, P. (1889). L'automatisme psychologique. Paris: Felix Alcan.

Kluft, R. P. (1984). An introduction to multiple personality disorder. Psychiatric Annals, 14(1), 19-24.

Kluft, R. P. (1988). On treating the older patient with multiple personality disorder: "Race against time" or "make haste slowly." American Journal of Clinical Hypnosis, 30(4), 257-266.

Kluft, R. P. (1999). Current issues in dissociative identity disorder. Journal of Psychiatric Practice, 1(5), 3-19.

Manfield, P., Lovett, J., Engel, L., \& Manfield, D. (2017). Use of the flash technique in EMDR therapy: Four case examples. Journal of EMDR Practice and Research, 11(4), 195-205.

Parnell, L. (2013). Attachment-focused EMDR. Healing relational trauma. New York, NY: W. W. Norton \& Company.

Paulsen, S. (1995). Eye movement desensitization and reprocessing: Its cautious use in the dissociative disorders. Dissociation, 8(1), 32-44.

Paulsen, S. (2008). Treating dissociative identity disorder with EMDR, ego state therapy, and adjunct approaches. In C. Forgash \& M. Copeley (Eds.), Healing the heart of trauma and dissociation with EMDR and ego state therapy (pp.141-179). New York, NY: Springer.

Prince, M. (1909). My life as a dissociated personality. Prince, M. (Ed.). Boston, MA: R. G. Badger.

Prince, W. F. (1917). The Doris case of quintuple personality. Journal of Abnormal Psychology, 11, 73-122.

Putnam, F. W. (1989). Diagnosis and treatment of multiple personality disorder. New York, NY: The Guildford Press.

Ross, C. A. (2015). When to suspect and how to diagnose dissociative identity disorder. Journal of EMDR Practice and Research, 9(2), 114-120.

Shapiro, F. (1995). Eye movement desensitization and reprocessing: Basic principles, protocols and procedures. New York, NY: Guildford Press.

Shapiro, F. (2018). Eye movement desensitization and reprocessing (EMDR) therapy, Third Edition: Basic principles, protocols, and procedures. New York, NY: The Guildford Press. 
Spiegel, H. (1972). An eye-roll test for hypnotizability. American Journal of Clinical Hypnosis, 15(1), 25-28.

Steele, K., Boon, S., \& Van der Hart, O. (2017). Treating trauma-related dissociation: A practical, integrative approach. New York, NY: W. W. Norton \& Company.

Van der Hart, O., Steele, K., Boon, S., \& Brown, P. (1993). The treatment of traumatic memories: Synthesis, realization, and integration. Dissociation, 6(2-3) 162-180.

Van der Hart, O., Nijenhuis, E. R. S., \& Steele, K. (2006). The haunted self: Structural dissociation and the treatment of chronic traumatization. New York, NY: W. W. Norton \& Company. 